\title{
Computer Program Calculation for Distortion of Wide-Band Track and Hold Amplifier
}

\author{
Hailang Liang ${ }^{1,2}$, Jin $\mathrm{He}^{1,2}$, Xiaoan Zhü ${ }^{2,3}$, Xiaomeng $\mathrm{He}^{2}$, Cheng Wang ${ }^{2,3}$, Lin $\mathrm{He}^{2}$, Gui Liư ${ }^{2}$, \\ Qingxing $\mathrm{He}^{4}$, Caixia $\mathrm{Du}^{4}$
}

${ }^{1}$ Tera-Scale Research Center, Institute of Microelectronics, School of Electronic Engineering and Computer Science, Peking University, Beijing 100871, China; ${ }^{2}$ Peking University Shenzhen SOC Key Laboratory, PKU-HKUST Shenzhen-Hong Kong Institution, Shenzhen 518057, China; ${ }^{3}$ Dong Guan Xin Cheng Electronic Technology Ltd. Co., High-Tech Industrial Development Zone, Songshanhu Lake, Dongguan, China; ${ }^{4}$ Shenzhen Huayue Terascale Chip Ltd. Co., Shenzhen 518045, China

Email: frankhe@pku.edu.cn

Received June 2013

\begin{abstract}
Tow different computer calculation methods for distortion of the wide-band diode bridge track and hold amplifier (THA) are presented based on a high frequency Schottky diode model. One of the computer programs calculates the distortion of weekly nonlinear THA based on the KCL and the nonlinear-current method. The other calculates the weekly nonlinear distortion by using a Volterra series method and a nodal formulation. Comparative calculation results for the diode bridge THA have shown good agreement with these two computer program calculation methods, whereas the overall computational efficiency of the nonlinear-current method is better than that of the nodal formulation method in a special evaluation.
\end{abstract}

Keywords: Track and Hold Amplifier; Broadband Amplifiers; High-Speed Integrated Circuits; Schottky Diode Frequency Converters; Harmonic Distortion; Volterra Analysis; Computer Program; Nonlinear-Current Method

\section{Introduction}

A diode bridge architecture THA prior to the analog to digital converters are used to implement low-cost ultra high speed transceivers. Distortion is one of the important factors that limit the dynamic range of THA [1]. It is crucial to predict and calculate the distortion in the weakly nonlinear circuit by computer program calculation or simulation. Several methods used to resolve the distortion issues are shown in [2-6]. Two computer programs based on these methods are implemented to evaluate the distortion of THA with an improved procedure in terms of the Volterra series.

In this paper, two computer program are used to calculate the weakly nonlinear distortion of the diode bridge switch THA based on the high frequency Schottky diode model in terms of a Volterra-series analysis. Section 2 describes a diode bridge THA for ultra high speed application. In Section 3, the high speed diode model, assumptions and distortion analysis are presented. The computer program description is proposed in Section 4. Section 5 shows the program calculation results and then follows by conclusion.

\section{A Diode Bridge THA}

Consider a diode bridge THA shown in Figure 1, which is directly derived from Figure 2 in [7], this circuit consists of a high-speed Schottky diode bridge ( $D_{1}$ to $D_{4}$ ) used as a switch between the input and output, a hold

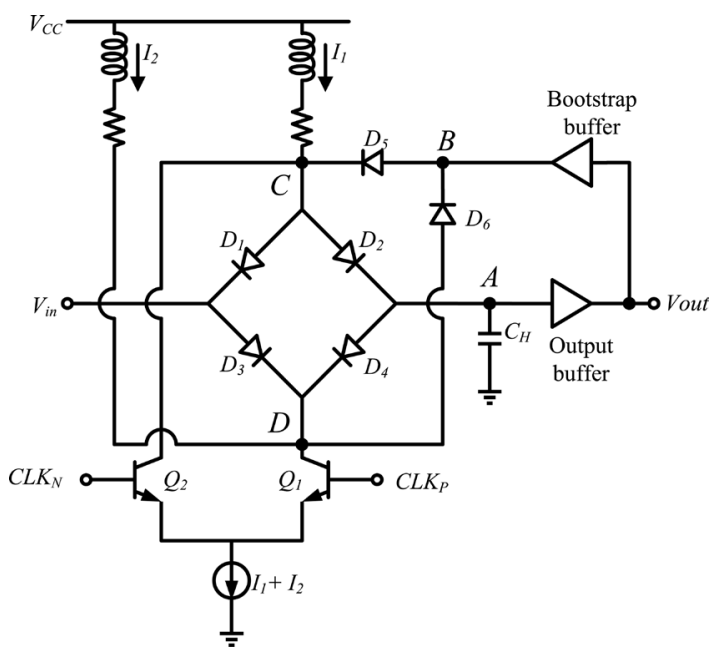

Figure 1. A diode bridge track and hold amplifier [7]. 


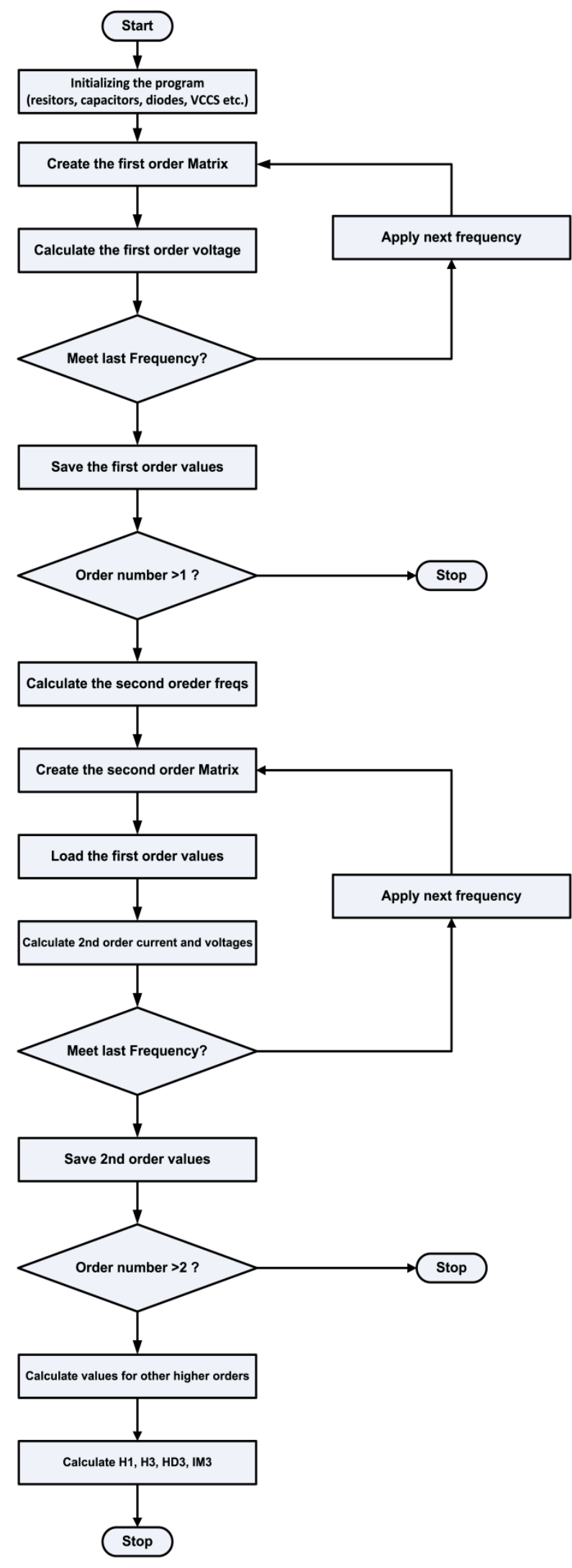

Figure 2. Program flow chart for calculating the distortion $[4,10]$. capacitor $C_{H}$ to maintain the sampling voltage, a differential pair ( $Q_{1}$ and $Q_{2}$ ) to turn on/off the diode bridge switch, two load inductors used to extend bandwidth, two load resistors, a bootstrap buffer, a output buffer and a current tail. This THA with diode bridge configuration is suitable for wideband and high speed application [7].

\section{Model, Assumptions and Distortion Analysis}

As shown in [8]. A simplified equivalent circuit for the diode bridge THA in track mode is illustrated in Figure 3(a).

The first order Volterra kernel $H_{1}\left(j \omega_{1}\right)$ is given by the following Equation (1) [1].

$$
H_{1}\left(j \omega_{1}\right)=\frac{\frac{1}{r_{d}}+j \omega_{1} C_{K}}{j \omega_{1} C_{H}+\frac{1}{r_{d}}+j \omega_{1} C_{K}}
$$

The second order and the third Volterra kernel $H_{2}\left(j \omega_{1}, j \omega_{2}\right)$ and $H_{3}\left(j \omega_{1}, j \omega_{2}, j \omega_{3}\right)$ are shown in Equation (2) and Equation (3) respectively. Equation (2) and Equation (3) imply that if $H_{1}\left(j \omega_{1}\right)=1$, the Volterra kernels $H_{2}\left(j \omega_{1}, j \omega_{2}\right)$ and $H_{3}\left(j \omega_{1}, j \omega_{2}, j \omega_{3}\right)$ tend to zero.

$$
\begin{gathered}
H_{2}\left(j \omega_{1}, j \omega_{2}\right)=\frac{-\frac{I_{d}}{2 V_{t}^{2}}\left(1-H_{1}\left(j \omega_{1}\right)\right)^{2}}{-\frac{I_{d}}{V_{t}}+\frac{1}{r_{d}}+C_{K}\left(j \omega_{1}+j \omega_{2}\right)} \\
H_{3}\left(j \omega_{1}, j \omega_{2}, j \omega_{3}\right)=\frac{\frac{I_{d}}{V_{t}^{2}} H_{2}\left(j \omega_{1}, j \omega_{2}\right)\left(H_{1}\left(j \omega_{1}\right)-1\right)}{\frac{I_{d}}{V_{t}}-\frac{1}{r_{d}}-C_{K} j\left(\omega_{1}+\omega_{2}+\omega_{3}\right)} \\
+\frac{I_{d}}{6 V_{t}^{3}}\left(1-3 H_{1}\left(j \omega_{1}\right)+3 H_{1}^{2}\left(j \omega_{1}\right)-H_{1}^{3}\left(j \omega_{1}\right)\right) \\
\frac{I_{d}}{V_{t}}-\frac{1}{r_{d}}-C_{K} j\left(\omega_{1}+\omega_{2}+\omega_{3}\right)
\end{gathered}
$$

The third-order harmonic distortion $\mathrm{HD}_{3}$ is the ratio of the amplitude of the third-order harmonic to the amplitude of the fundamental harmonic. With the Volterra coefficients, $\mathrm{HD}_{3}$ is defined as followings [1,9].

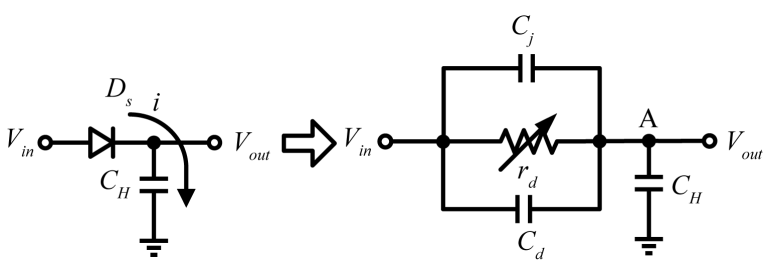

(a)

(b)

Figure 3. A simplified equivalent circuit for the diode bridge THA. 


$$
H D_{3}=\frac{1}{4} \frac{\left|H_{3}\left(j \omega_{1}, j \omega_{2}, j \omega_{3}\right)\right|}{\left|H_{1}\left(j \omega_{1}\right)\right|} \circ V_{i n}^{2}
$$

The third-order intermodulation distortion which is the ratio of third-order kernel to the first-order kernel can be expressed as [1].

$$
I M_{3}=\frac{3}{4} \frac{\left|H_{3}\left(\omega_{1}, \omega_{2}, \omega_{3}\right)\right|}{\left|H_{1}\left(\omega_{1}\right)\right|^{3}} \circ V_{i n}^{2}
$$

In term of Volterra analysis, one can choose the appropriate values for the bias current and the hold capacitor, $C_{H}$, to yield a suitable $I M_{3}$ performance [1].

\section{Computer Program Description}

(1) Nonlinear-current method:

The KCL and the nonlinear-current method are used in this program. The detailed description of the nonlinearcurrent method was proposed in [5,6], [4] and [10]. In this approach the small-signal I/V characteristic for a simple voltage-controlled conductance is expressed as [10]:

$$
i=g_{1} v+g_{2} v^{2}+g_{3} v^{3}+g_{4} v^{4}+g_{5} v^{5}+\ldots
$$

where the first-order voltage $v(t)$ is

$$
v(t)=\frac{1}{2} \sum_{q=-Q}^{Q} v_{s, q} \exp \left(j \omega_{q} t\right)
$$

the second-order current $[4,10]$ is

$$
\begin{aligned}
& i_{2}(t)=g_{2} v_{1}^{2}(t) \\
& +\frac{g_{2}}{4} \sum_{q_{1}=-Q q_{2}=-Q}^{Q} \sum_{1, q_{1}}^{Q} v_{1, q_{2}} \exp \left[j\left(\omega_{q_{1}}+\omega_{q_{2}}\right) t\right]
\end{aligned}
$$

This program is implemented in Python in terms of the nonlinear-current method and KCL $[4,10]$. The linear resistors, diodes, capacitors, inductors, controlled sources, transmission lines and the nonlinear resistors, diodes, capacitors, and controlled sources are used in this program for calculating the distortion of Volterra series. The flow chart for the program is illustrated in Figure 2, where the basic algorithm used in this paper is depicted in $[4,10]$.

The program first initializes the environmental variables, which includes setting up the values of the resistors, the capacitors, the diodes, the inductors, the VCCS etc. and replacing every non-linearity with its linearized equivalence.

Then the program begins to calculate the first order voltage with linearized equivalence [4]. The output data are saved and then the second and the third order currents and voltages are computed, the calculation of these higher order currents and voltage depends on those of the lower order ones that have been saved. As shown in the flow chart, the related currents and voltages are solved for each order and the desired corresponding responses are derived from the Volterra series.

(2) Volterra series and nodal formulation method:

The flow chart of this method is shown in Figure 4. More detailed description of this method is depicted in [11]. To compare with the results from those of nonlinear-current method, the program has been rewritten in Python language.

\section{Calculation Results}

Figure 5 illustrates the IIP3 versus input frequency results of both nonlinear-current method and nodal formulation method, which indicates that these two results have a good agreement in the range of the input frequency.

The results of the $H D_{3}$ versus input frequency for both nonlinear-current method and nodal formulation method

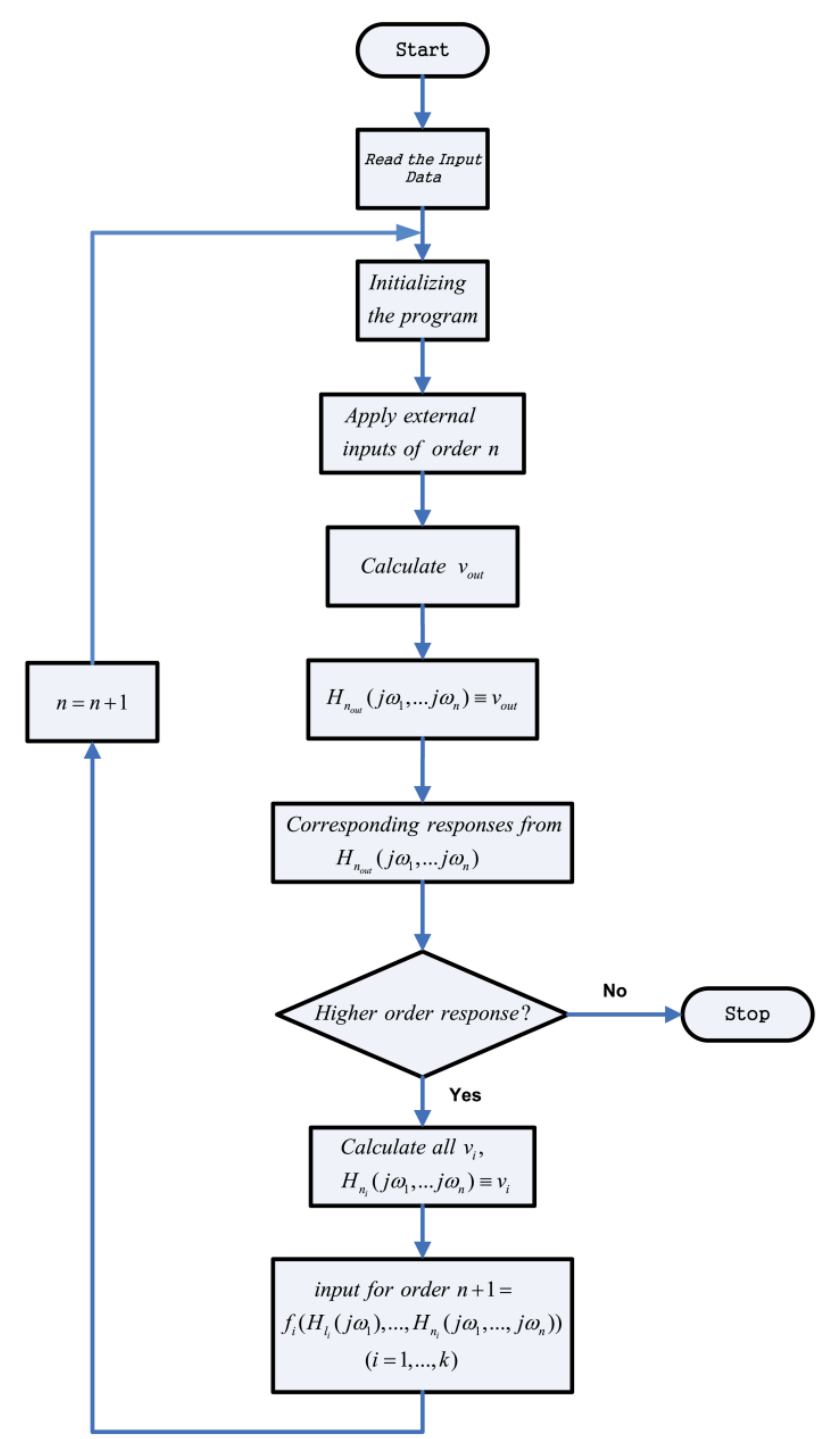

Figure 4. Program flow chart for the computation of volterra kernels [2]. 


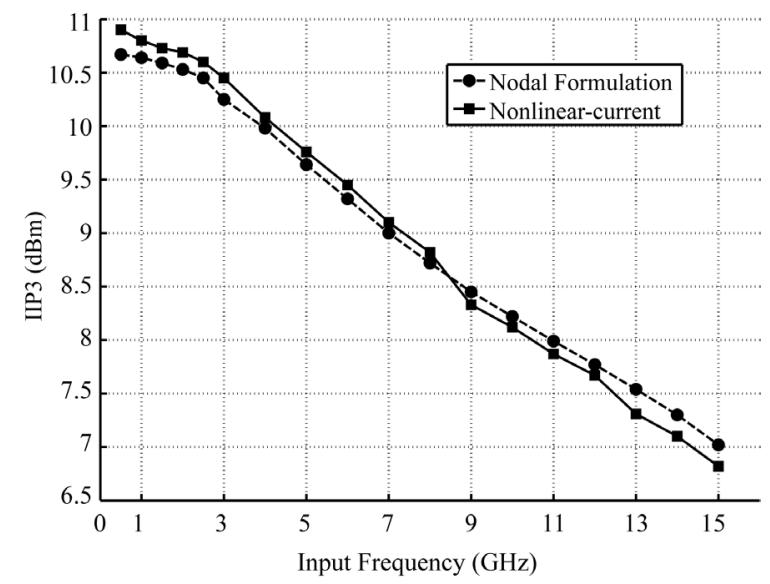

Figure 5. Comparison of third-order intercept point.

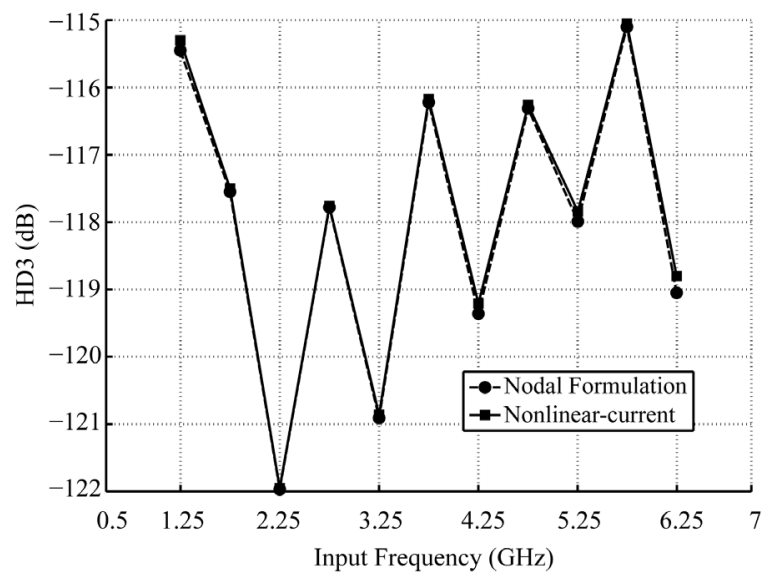

Figure 6. $H D_{3}$ characteristics of diode bridge THA.

are illustrated in Figure 6, it shows that they are also similar and have the agreement between them.

At some special test condition, the calculation time results of both nonlinear-current method and nodal formulation method are illustrated in Table 1. Obviously, the calculation times of the nonlinear-current method is much less than those of nodal formulation method with the specified input frequency.

\section{Conclusion}

Nonlinear-current method and nodal formulation method for distortion calculation of the diode bridge configuration THA are presented by using a simplified high-speed diode model. Comparative results show that the calculation results derived by the nonlinear-current method is consistent with those of the nodal formulation method, whereas the overall calculation time of the nonlinearcurrent method has been improved.

\section{Acknowledgements}

This work is supported by the Industry Education and
Table 1. Summary of calculation time.

\begin{tabular}{cccc}
\hline Frequency $(\mathrm{GHz})$ & 1 & 3 & 5 \\
\hline Nonlinear-current method (Sec) & 12.78 & 36.42 & 42.75 \\
Nodal formulation method (Sec) & 25.17 & 72.49 & 87.73 \\
\hline
\end{tabular}

Research Foundation of PKU-HKUST Shenzhen-Hongkong Institution (sgxcyhzjj201204), by the Guangdong Natural Science Foundation (S2011040001822) and the Fundamental Research Project of Shenzhen Science and Technology Foundation JCYC20120618163025041. This work is also supported by the National natural Science Funds of China (61204033, 61204043).

\section{REFERENCES}

[1] J. C. Jensen, "Ultra-High Speed Data Converter Building Blocks in Si/SiGe HBT Process," Ph.D. Dissertation, University of California, San Diego, 2005.

[2] P. Wambacp and W. Sansen, "Distortion Analysis of Analog Integrated Circuits,” 1em plus 0.5em minus 0.4em, Kluwer, Norwell, MA, 1998. http://dx.doi.org/10.1007/978-1-4757-5003-4

[3] J. Vuolevi and T. Rahkonen, Distortion in RF Power Amplifiers. 1em plus 0.5em minus 0.4em Artech House INC., Norwood, MA, USA, 2003.

[4] D. D. Weiner and J. E. Spina, "Sinusoidal Analysis and Modeling of Weakly Nonlinear Circuits,” 1em plus 0.5em minus 0.4em, Van Nostrand, New York, 1980.

[5] S. Maas, "Nonlinear Microwave Circuits," 1em plus 0.5em minus 0.4em Artech House, Norwood, MA, 1988.

[6] J. Bussgang, L. Ehrman and J. Graham, "Analysis of Nonlinear Systems with Multiple Inputs,” Proceedings of the IEEE, Vol. 62, No. 8, 1974, pp. 1088-1119. http://dx.doi.org/10.1109/PROC.1974.9572

[7] J. Jensen and L. Larson, "A Broadband 10 GHz Trackand-Hold in Si/SiGe HBT Technology," Proceedings of the IEEE 2000 Custom Integrated Circuits Conference, 2000, pp. 245-248.

[8] H. Liang, R. Evans and E. Skafidas, "Distortion Analysis of Ultra Wide-Band Diode Bridge Track and Hold Amplifier," 53rd IEEE International Midwest Symposium on Circuits and Systems (MWSCAS), 2010, pp. 721-724.

[9] R. Minasian, "Intermodulation Distortion Analysis of MESFET Amplifiers Using the Volterra Series Representation," IEEE Transactions on Microwave Theory and Techniques, Vol. 28, No. 1, 1980, pp. 1-8. http://dx.doi.org/10.1109/TMTT.1980.1129998

[10] S. Maas, “A General-Purpose Computer Program for the Volterra-Series Analysis of Nonlinear Microwave Circuits,” IEEE MTT-S International Microwave Symposium Digest, Vol. 1, 1988, pp. 311-314.

[11] H. Liang, J. He, C. Wang, X. Zhu and M. Chan, “A MATLAB Program for Volterra Distortion Analysis in CMOS Switched Source Follower," IEEE Asia Pacific Conference on Circuits and Systems (APCCAS), 2012, pp. 108-111. 